\title{
Can we distinguish Majorana and Dirac neutrinos in solar neutrino experiments?
}

\author{
V.B. Semikoz 円 \\ The Institute of the Terrestrial Magnetism, the Ionosphere and Radio Wave Propagation \\ of the Russian Academy of Sciences, \\ IZMIRAN, Troitsk, Moscow region, 142092, Russia
}

\begin{abstract}
If neutrino conversions within the Sun result in partial polarization of initial solar neutrino fluxes then a new opportunity to distinguish Majorana and Dirac neutrinos by measuring the differential $\nu_{e} e$-scattering cross section in solar neutrino detectors arises.

The experiment like HELLAZ would be preferable in testing of recoil electron spectra differences initiated by Majorana and Dirac neutrinos since low energy recoil electrons $(T \geq 100 \mathrm{keV})$ can be detected and electron energy and direction can be determined with good precision.
\end{abstract}

PACS codes: 13.10.+q; 13.15.-f; 13.40.Fn; 14.60.Gh; 96.60.Kx.

Key words: neutrino, magnetic moment, magnetic fields, polarization.

\footnotetext{
${ }^{1}$ E-mail: semikoz@charley.izmiran.rssi.ru", fax: 7-095-3340124
} 


\section{Introduction}

Lower bounds on neutrino masses have not been found yet in direct laboratory experiments. In particular, in the case of neutrinoless double-beta decay one expects a decrease of an upper bound on the Majorana mass $m_{\nu_{e}}^{(M)}$ only. This current improvement of upper limits on neutrino masses takes a long time and strong efforts. However, we can not justify on this way whether neutrino is really the Majorana particle or it can be the Dirac one, or a mixture of them (ZKM-neutrino).

In this connection let us recall the old experiment by Davis [1] who demonstrated that neutrino and antineutrino are indeed different particles if we are using $\tilde{\nu}_{e}$ from the beta-decay $n \rightarrow p e^{-} \tilde{\nu}_{e}$ as the incident "neutrino" for the capture process $\nu_{e}+{ }^{37} \mathrm{Cl} \rightarrow$ ${ }^{37} \mathrm{Ar}+\mathrm{e}^{-}$.

Negative result of the experiment[1], $\nu_{e} \neq \tilde{\nu}_{e}$, is not an evidence that $\nu_{e}$ and $\tilde{\nu}_{e}$ are the Dirac neutrinos with the fixed lepton number $L=\mp 1$. In such experiments the helicity $r=\mp 1$ (upper signs for $\nu_{e}$ ) is appropriate quantum number which is conserved due to the $V-A$ law of charged current weak interaction rather the lepton number.

Both the right-handed Majorana neutrino and the Dirac antineutrino with the same helicity $r=+1$ could be emitted in the beta decay $n \rightarrow p e^{-} \tilde{\nu}_{e}$ with the following suppression of the spin-flip $r=+1 \rightarrow r=-1$ in the process of capture in the chlorine detector (the latter is true to the order of $O\left(\left(m_{\nu} / E\right)^{2}\right) \ll 1$ in the cross section).

Thus this example demonstrates the well-known fact that in the massless limit $m_{\nu} \rightarrow 0$ Majorana and Dirac neutrinos are not distinguishable. We can not mark any difference between the fully-polarized right-handed Majorana neutrino $\tilde{\nu}_{e_{R}}$ and the right -handed Dirac antineutrino $\tilde{\nu}_{e}^{(D)}$ as well as between the left-handed Majorana neutrino $\nu_{e_{L}}$ and the Dirac left-handed one, $\nu_{e_{L}}=\nu_{e_{L}}^{(D)}$ (see below section 3).

In turn, if an incident neutrino flux became partially-polarized this would give a chance to distinguish these particles.

We propose here a new way for distinction of Majorana and Dirac neutrino in the solar neutrino experiments by studying the profiles of the electron spectra in the low-energy $\nu_{e} e$-scattering for incident $\nu^{(M)}$ and $\nu^{(D)}$ fluxes. It seems possible when solar neutrino flux is partially-polarized.

The ultrarelativistic neutrinos produced in thermonuclear reactions within solar interior are evidently the left-handed ones (fully-polarized $\nu_{e_{L}}$ ) and one needs to assume some mechanism for their conversion to the right-handed neutrinos.

First, the conversions $\nu_{e_{L}} \rightarrow \tilde{\nu}_{e_{R}}$ in the Majorana case or $\nu_{e_{L}} \rightarrow \nu_{e_{R}}$ in the Dirac case are obviously based on the assumption of a non-vanishing neutrino mass $m_{\nu} \neq 0$. This leads to nonzero neutrino diagonal [2] and transition [3] dipole moments and, therefore, to the possible neutrino spin [2] and spin-flavor precession [4] in vacuum in the presence of an external magnetic field. In a medium neutrino oscillations and spin-flavor precession can occur as the resonant conversions $\nu_{e_{L}} \rightarrow \nu_{\mu_{L}}$ [5] and $\nu_{e_{L}} \rightarrow \tilde{\nu}_{\mu_{R}}[6]$. 
The spin-flavor conversion in combination with the MSW-mechanism can lead to the right-handed Majorana neutrino production $\left(\nu_{e_{L}} \rightarrow \tilde{\nu}_{e_{R}}\right.$ [7], see below section 2 ), i.e. to a mixture of the left-and right-handed active neutrinos as a partiallypolarized $\nu_{e_{L}}, \tilde{\nu}_{e_{R}}$ neutrino flux incident upon underground detectors. In contrast to the Majorana neutrino case, for the same conditions in the Sun the right-handed Dirac neutrinos produced via the spin-flip $\nu_{e_{L}} \rightarrow \nu_{e_{R}}$ or in the cascade conversions $\nu_{e_{L}} \rightarrow \nu_{\mu_{R}} \rightarrow \nu_{e_{R}}$ appear to be sterile ones with respect to the $\nu_{e} e$-scattering in detectors.

Notice that necessary large values of transition moments (even without direct proportionality to $m_{\nu}$ ) can be obtained in some extended models obeying all known laboratory, astrophysical and cosmological constraints on neutrino masses and on its dipole moments.

For all Majorana and Dirac neutrinos with a mass below $5 \mathrm{keV}$ the most restrictive limit on dipole or transition magnetic and electric moments $\mu_{\nu} \lesssim 3 \times 10^{-12} \mu_{B}$ arises from the absence of anomalous neutrino emission from the red-giant cores just before helium ignition [8]. The condition $m_{\nu} \lesssim 5 \mathrm{keV}$ follows from a kinematic limit on the neutrino energy $E_{\nu_{a, b}}$ lost in the plasmon decay $\gamma^{*} \rightarrow \nu_{a} \tilde{\nu}_{b}$ since the plasma frequency in a degenerate electron gas of red- giants is bounded in the same region, $\omega_{p} \lesssim 5-10 \mathrm{keV}$.

A large Dirac neutrino magnetic moment (both diagonal and transition ones, $\left.\mu_{\nu}^{(D)} \gtrsim 3 \times 10^{-12} \mu_{B}\right)$ was also excluded from SN1987A neutrino events in the

Kamiokande and IMB detectors. This is due to non-observation there of a hard energy tail stipulated by the sterile $\nu_{e_{R}}$ emission from a hot supernova core 9. These neutrinos could be produced within core via the electromagnetic scattering (see below Eq. (14)) and then be converted to the active $\nu_{e_{L}}$ in the intergalactic magnetic field .

The absence of SN1987A constraint in the Majorana case means that the modeldependent estimate of $\mu_{e \mu}$ [8] seems to be less consistent even for light neutrinos suitable for the resonant spin-flavor or the MSW conversions in the Sun.

Therefore the laboratory constraint from reactor antineutrino experiments which is common for diagonal and transition magnetic moments, $\mu_{\nu_{e}} \lesssim 2-4 \times 10^{-10} \mu_{B}$ [10], remains an upper estimate of Majorana neutrino transition moments corresponding to effective neutrino conversions within solar convective zone with magnetic fields of order $\sim 1 k G$.

On the other hand, in magnetic hydrodynamics one can not exclude solutions with a strong magnetic field near bottom of the convective zone of the Sun, $\sim 100 k G[11$, and even larger within solar core for equilibrium hydromagnetic configuration in the gravitational field, $B \lesssim 10^{8} G[12]$. As a result even for the case when the limit $\mu_{e \mu} \lesssim 3 \times 10^{-12} G[\mathbb{8}]$ is valid we may apply some mechanisms for effective spin-flavor conversions.

Notice also that the most stringent constraints on transition magnetic moments $\mu_{e s} \lesssim 10^{-16} \mu_{B}$ were derived in 13 from the primordial nucleosynthesis bound on additional neutrino species and from a supernova energy loss argument. These bounds 
were obtained in [13] for active-sterile neutrino conversions neglecting neutrino mixing and in a medium with random magnetic fields. Therefore this approach has no relation to our consideration here since we are using the models [6, 7] for active-active neutrino conversions.

In the end of section 2 we consider experimental bounds on $\tilde{\nu}_{e_{R}}$ and give a theoretical interpretation of some contradictions in the model used. In particular, we consider an important question how to avoid some known lacks of the spin-flavor scenario [7] with the use of large neutrino magnetic moments that inevitably leads to contradiction with non-observation of temporal variations of the solar neutrino flux in the most of current experiments.

Then in section 3 we show why the scattering of partially-polarized neutrino flux off electrons should be absolutely different for Majorana and Dirac neutrino provided that their right-handed components interact (do not interact in the Dirac case) with electrons.

In section 4 we discuss results and crucial parameters of the model used.

\section{Mechanisms of the right-handed Majorana and Dirac neutrinos production in the Sun.}

The main assumption here is the presence of the right-handed neutrinos $\tilde{\nu}_{e_{R}}, \nu_{e_{R}}$ produced by some mechanism within the Sun. For instance, in the spin-flavor scenario involving Majorana neutrinos the Sun would be a source for antineutrinos, some of which could be $\tilde{\nu}_{e_{R}}$ 's by a combination of spin-flavor $\left(\nu_{e_{L}} \rightarrow \tilde{\nu}_{\mu_{R}}\right)$ and flavor $\left(\tilde{\nu}_{\mu_{R}} \rightarrow\right.$ $\tilde{\nu}_{e_{R}}$ ) oscillations (or due to permutation of steps above, $\nu_{e_{L}} \rightarrow \nu_{\mu_{L}} \rightarrow \tilde{\nu}_{e_{R}}$ ). This scenario was in detail elaborated in [7] for the case of twisting magnetic field that allowed authors to avoid the suppression of the cascade conversion $\nu_{e_{L}} \rightarrow \tilde{\nu}_{e_{R}}$ caused by the almost full compensation of the partial (two-step) amplitudes, $M_{1}+M_{2} \approx 0$, (for a small neutron abundance in the Sun).

Moreover, authors [7] found conditions when either $\nu_{e_{L}}-\tilde{\nu}_{e_{R}}$-system decouples from $\nu_{\mu_{L}}$ and $\tilde{\nu}_{\mu_{R}}$ or the triple resonances merge and a resonant transition leads to a complete $\nu_{e_{L}} \rightarrow \tilde{\nu}_{e_{R}}$-conversion. In the case of separation of $\nu_{e_{L}}-\tilde{\nu}_{e_{R}}$-system the adiabaticity condition is not fulfilled (at least for the convective zone in the Sun) while for the triple resonance the relevant adiabaticity parameters at the merging point depend on neutrino energy.

As result authors [7] predict that the $\tilde{\nu}_{e_{R}}$-flux should have a peak at the energy $E$ defined by the resonant condition. Explicitely this occurs for $\dot{\Phi}<0$ at $\left(s_{2} \delta\right) \simeq \mu B_{\perp}$, where $s_{2}=\sin 2 \theta$ is the mixing parameter; $\delta=\Delta m^{2} / 4 E$ and $\Delta m^{2}>0$ is the neutrino squared mass difference; $\mu$ is the neutrino transition magnetic moment; $B_{\perp}$ is the amplitude of the twisting magnetic field $\mathbf{B} \sim e^{i \Phi(t)}$ which is perpendicular to the momentum $\mathbf{k}$ of an ultrarelativistic neutrino, $E \approx k$.

Since the position and the width of the peak strongly depend on the magnetic field 
strength in the region of resonance, the energy spectrum of resonantly emitted solar $\tilde{\nu}_{e_{R}}$ 's should exhibit characteristic time dependence. In particular, depending on the signs of $\dot{\Phi}$ in the northern and southern solar hemispheres the semiannual neutrino flux variation? can be either enhanced or suppressed. Moreover, the energy at which the $\tilde{\nu}_{e_{R}}$-spectrum achieves the maximum should vary in time .

Note also other ways of the Majorana $\tilde{\nu}_{e}$-production like $\nu_{e} \rightarrow \tilde{\nu}_{e}+\chi$-decay (to majoron) enhanced in solar matter [15]. In this case we do not need any strong magnetic field in the Sun or the presence of a large neutrino magnetic moment. However, the $\tilde{\nu}_{e}$-spectrum differs in such case from the initial one for left-handed neutrinos that prevents from the approach developed below.

The active Dirac antineutrino $\tilde{\nu}_{e_{R}}=\tilde{\nu}_{e}^{(D)}$ never can be produced in the Sun due to absence there of the annihilation process $e^{-} e^{+} \rightarrow \nu \tilde{\nu}$ which is important, for instance, in a supernova. However, in the same twisting magnetic field the resonant $\nu_{e_{L}}^{(D)} \rightarrow$ $\nu_{e_{R}}^{(D)}$-conversions lead to the production of the sterile neutrinos $\nu_{e_{R}}^{(D)}$ through a diagonal magnetic moment $\mu_{\nu}[\mathbb{1 6}]$ ( neglecting neutrino flavor mixing) or analogously to the Majorana case in the presence of mixing and via a transition magnetic moment for the system of the active $\nu_{e_{L}}^{(D)}, \nu_{\mu_{L}}^{(D)}$ neutrinos and their sterile $\nu_{e_{R}}^{(D)}, \nu_{\mu_{R}}^{(D)}$ - components.

On the other hand, it is well-known that the Kamiokande data already yield restrictive limits on a solar $\tilde{\nu}_{e_{R}}$-flux at the high energy region, $E_{\nu} \gtrsim 8.5 \mathrm{MeV}$ [17]. This limit was found from an isotropic background accounting also for $\tilde{\nu}_{e_{R}}$ 's due to the relatively large cross-section $\sigma\left(\tilde{\nu}_{e} p \rightarrow n e^{+}\right) \sim 9.4 \times 10^{-44} \mathrm{~cm}^{2}\left(p_{e} E_{e} / M e V^{2}\right)$ with the isotropic distribution of positrons, where $E_{e}=E_{\nu}-1.3 \mathrm{MeV}$. The best relative limit is at $E_{\nu}=13 \mathrm{MeV}$ where the $\tilde{\nu}_{e_{R}}$ flux is less than $5.8 \%$ of ${ }^{8} \mathrm{~B}$ solar $\nu_{e}$ 's at $90 \%$ CL.

However, since the $\nu_{e_{L}} \rightarrow \tilde{\nu}_{e_{R}}$-conversion within the Sun depends on neutrino energy, sign of $\dot{\Phi}$, etc., and due to the absence of the Kamiokande limit for such process below $E_{\nu} \lesssim 8 \mathrm{MeV}$ we can not exclude the presence of right-handed Majorana neutrinos emitted by the Sun, in particular, for the low-energy beryllium neutrinos (the line $E_{\nu_{e}}=0.862 \mathrm{MeV}$ ). One expects to observe these neutrinos in the Borexino (1998?) and the Hellaz experiments where a scintillation detector [18] and a helium gas at azote temperature 19 should have a low-energy threshold.

Notice that for the beryllium neutrino line emitted by the Sun the reaction $\tilde{\nu}_{e} p \rightarrow$ $n e^{+}$vanishes because of its high threshold $E_{\nu t h}=1.8 \mathrm{MeV}$, but the parallel branch$\tilde{\nu} e$-scattering- contributes to the counting of neutrino events starting from a detector threshold.

In anyway, since we assume here the right-handed neutrinos are producing in the

\footnotetext{
${ }^{2}$ First these semiannual variations were predicted in 国 for the neutrino magnetic moment interaction with the constant toroidal magnetic field (azimuthal component) which changes sign at the solar equator. Inclination of the ecliptic (of the Earth orbital plane) with respect to solar equator $\left(7^{\circ} 15^{\prime}\right)$ leads to the minimum of the neutrino interaction term $\mu B_{\perp}$ for the two season positions of the Earth (in June and December) when the solar core is viewed from the Earth through the solar equator.
} 
Sun and the first scenario [7] realizes, there appear some known objections concerning a large value $\mu B_{\perp}$ for an effective neutrino spin reversal or non-observation of the semiannual flux variations in the current neutrino experiments.

Really, from the astrophysical bound on any Dirac and Majorana neutrino magnetic moments $\mu_{\nu} \lesssim 3 \times 10^{-12} \mu_{B}$ [8] it follows that necessary neutrino spin reversal when the dimensionless parameter $\mu_{\nu} B_{\perp} L$ should be of the order unity, $\mu_{\nu} B_{\perp} L \sim 1$, is provided by the sufficiently strong magnetic fields $B \sim 100 k G$ applied along a half of the width of the convective zone, $L \sim 10^{10} \mathrm{~cm}$. Even such magnetic fields would not be in contrary with some estimates in magnetic hydrodynamics [11, 12]. Meanwhile, a more weakened and model-independent laboratory constraint $\mu_{12} \lesssim 10^{-10} \mu_{B}-$ $-10^{-11} \mu_{B}$ [10 seems to be reasonable for a Majorana neutrino for which the SN1987A energy loss argument [9] does not work in contrast to the Dirac case. This means that magnetic field strength in the Sun could be less than for the Dirac case, or quite realistic values $B \sim 10^{3}-10^{4} G$ would be sufficient for effective spin-flavor conversions.

Non-observation of the semiannual flux variations, perhaps, is explained either by unsuccessful sign of the angular velocity $\dot{\Phi}$ for the twisting magnetic field in both solar hemispheres (in mechanism [7] under consideration, see above)) or even more simpler than in [7] without any specific geometry of a magnetic field: by the presence of random magnetic fields which always exist in addition to a regular magnetic field and can have the same strength of the r.m.s. field $\sqrt{\left\langle\tilde{B}^{2}\right\rangle} \sim B$. In contrast to regular field, random magnetic fields do not disappear at the equatorial plane while $<\tilde{B}>=0$ elsewhere and their scale $L_{0}$ is much less than the regular field one.

Thus one can pronounce that experimental bounds at present do not exclude the possible observation of the low-energy $\tilde{\nu}_{e_{R}}$ 's in future solar neutrino experiments .

\section{Scattering of partially polarized neutrinos off electrons}

\subsection{Polarization density matrix}

Both right-handed Majorana (active) neutrinos and right-handed (sterile) Dirac neutrinos can influence the observed neutrino events but in different ways.

Really, below we find that for partially polarized electron neutrino, $\left|\xi_{z}^{(0 e)}\right|<1$, where

$$
\rho_{r r^{\prime}}=\frac{A \delta_{r r^{\prime}}+\xi_{i}^{(0 e)}\left(\sigma_{i}\right)_{r r^{\prime}}}{2}
$$

is the spin density matrix of the solar neutrino flux $\left(r, r^{\prime}= \pm 1\right)$ with the initial momentum directed along z-axis, $\mathbf{k}_{1}=\left(0,0, k_{1}\right)$, the differential cross-sections of weak interactions, $(d \sigma / d T)_{\text {weak }}$, are the different ones for Dirac and Majorana neutrinos.

The main point is that the difference of the cross-sections gives the different electron energy spectrum profiles for two kinds of neutrino. 
As well as in the case of a Dirac neutrino in [14] the polarization components of electron Majorana neutrinos in Eq. (11) are

$$
\begin{aligned}
& \xi_{z}^{(0 e)}=\tilde{\nu}_{e_{R}}^{*} \tilde{\nu}_{e_{R}}-\nu_{e_{L}}^{*} \nu_{e_{L}} \\
& \xi_{\perp}^{(0 e)}=2\left|\nu_{e_{L}}^{*} \tilde{\nu}_{e_{R}}\right|
\end{aligned}
$$

with the essential distinction that now the right-handed component $\tilde{\nu}_{e_{R}}$ is active.

The spin density matrix for the muon neutrinos is given by the same Eq.(11) with the change of the index $(0 e)$ to $(0 \mu)$ and of the subscripts $e L, e R$ to $\mu L, \mu R$ for the polarization components (2) .

Notice also that, in contrast to [14], the normalization of the electron spin density matrix Eq. (1), in general, is different from unity ,

$$
\operatorname{Tr} \rho=A=\tilde{\nu}_{e_{R}}^{*} \tilde{\nu}_{e_{R}}+\nu_{e_{L}}^{*} \nu_{e_{L}}=1-\nu_{\mu_{L}}^{*} \nu_{\mu_{L}}-\tilde{\nu}_{\mu_{R}}^{*} \tilde{\nu}_{\mu_{R}}
$$

Using Eq. (1) and summing over the helicities of the partially polarized initial neutrino flux coming from the Sun to the detector,

$$
\rho=\sum_{r, r^{\prime}= \pm 1} u_{\nu_{e}}^{r^{\prime}}\left(k_{1}\right) \bar{u}_{\nu_{e}}^{r}\left(k_{1}\right) \rho_{r r^{\prime}}
$$

we obtain in the ultrarelativistic limit $\omega_{1} \approx k_{1} \gg m_{\nu}$ the well-known density matrix in $4 \times 4$-representation (compare formula (D.54) in the book [20] for $\mathrm{A}=1$ )

$$
\rho \simeq \frac{1}{2}\left(A+\xi_{z}^{(0 e)} \gamma_{5}+\gamma_{5} \hat{\xi}_{\perp}^{(0 e)}\right) \hat{k}_{1}
$$

which allows us to calculate in the subsection 3.2 the different mean $\nu e$-scattering cross-sections averaged over solar neutrino spectrum, $\left\langle\sigma^{(M)}\right\rangle$ and $\left\langle\sigma^{(D)}\right\rangle$.

Recall that the index (0) refers to the rest frame of a particle, $a_{\mu}=\left(0, \vec{\xi}^{(0)}\right)$, or $\left|\overrightarrow{\xi^{(0)}}\right|$ is the Lorentz-invariant due to the normalization of the spin 4-vector $a_{\mu}=\operatorname{Tr}\left(\rho \gamma_{\mu} \gamma_{5}\right) / 2 m_{e}, a_{\mu} a^{\mu}=-\vec{\xi}^{(0)}{ }^{2}$, in the Mishel-Wightman density matrix $\rho=$ $\left(\hat{k}_{1}+m_{\nu}\right)\left(1+\gamma_{5} \hat{a}\right) / 2$ which transits to (4) for $A=1$ in the ultrarelativistic limit $m_{\nu} \rightarrow 0$ [20].

There are also two additional density matrices with the mixed flavors in the interference of the weak and electromagnetic amplitudes,

$$
\begin{aligned}
& \rho^{(\mu \tilde{e})}=\sum_{r, r^{\prime}} u_{\nu_{e R}}^{r}\left(k_{1}\right) \bar{u}_{\nu_{\mu L}}^{r^{\prime}}\left(k_{1}\right) \rho_{\left.r r^{\prime}\right)}^{(\mu \tilde{e})} \simeq \frac{1}{4} \hat{\xi}_{\perp}^{(\mu \tilde{e})} \hat{k}_{1}\left(1+\gamma_{5}\right), \\
& \rho^{(\tilde{\mu} e)}=\sum_{r, r^{\prime}} u_{\nu_{\mu R}}^{r}\left(k_{1}\right) \bar{u}_{\nu_{e L}}^{r^{\prime}}\left(k_{1}\right) \rho_{r r^{\prime}}^{(\tilde{\mu} e)} \simeq \frac{1}{4} \hat{\xi}_{\perp}^{(\tilde{\mu} e)} \hat{k}_{1}\left(1+\gamma_{5}\right),
\end{aligned}
$$

\footnotetext{
${ }^{3}$ We use the opposite definition of the longitudinal polarization to the choice in 14 , so that for fully polarized neutrinos we mean $\xi_{z}^{(0 e)}=-1$ (left-handed Majorana or Dirac neutrinos).
} 
where the transversal polarization values are given by

$$
\begin{aligned}
& \left|\vec{\xi}_{\perp}^{(\mu \tilde{e})}\right|=2\left|\nu_{\mu L}\right|\left|\nu_{e R}\right|=2 \sqrt{P_{1}(A-P)} \\
& \left|\vec{\xi}_{\perp}^{(e \tilde{\mu})}\right|=2\left|\nu_{e L}\right|\left|\nu_{\mu R}\right|=2 \sqrt{P\left(1-A-P_{1}\right)} .
\end{aligned}
$$

We have determined here the mixed transversal polarizations (6) as well as the electron neutrino polarization components $\xi_{z}^{(0 e)}=A-2 P,\left|\vec{\xi}_{\perp}^{(0 e)}\right|=2 \sqrt{P(A-P)}$ and

the muon neutrino ones $\xi_{z}^{(0 \mu)}=1-A-2 P_{1},\left|\vec{\xi}_{\perp}^{(0 \mu)}\right|=2 \sqrt{P_{1}\left(1-A-P_{1}\right)}$ entering Eq. (2) as the functions of the survival probability $P=\nu_{e_{L}}^{*} \nu_{e_{L}}$, of the left-handed muon neutrino probability $P_{1}=\nu_{\mu L}^{*} \nu_{\mu L}$ and of the normalization coefficient $A$ given by Eq. (3). All functions $P, P_{1}$ and $A$ depend on the neutrino energy $E$, the mixing parameters $\delta s_{2}$, the magnetic energy $\mu B_{\perp}$ and the matter density.

These functions are very complicated in the scenario[7]. For instance, the merging of the three transitions, $\nu_{e_{L}} \leftrightarrow \nu_{\mu_{L}}, \tilde{\nu}_{e_{R}} \leftrightarrow \nu_{\mu_{L}}, \tilde{\nu}_{e_{R}} \leftrightarrow \nu_{e_{L}}$, yields the large $\nu_{e_{L}} \leftrightarrow \tilde{\nu}_{e_{R}}{ }^{-}$ transition probability given by Eq. (48) in [7], $P_{\nu_{e_{L}} \rightarrow \tilde{\nu}_{e_{R}}}=A-P$, which after the averaging over fast oscillations gives

$$
P_{\nu_{e_{L}} \rightarrow \tilde{\nu}_{e_{R}}}=\simeq \sin ^{2} \frac{4\left(s_{2} \delta\right)^{2}\left(\mu B_{\perp}\right)^{2}}{\left[\left(s_{2} \delta\right)^{2}+\left(\mu B_{\perp}\right)^{2}\right]^{2}} .
$$

For the beryllium line $E_{0}=0.862 \mathrm{MeV}$ the averaging of the cross-sections with the help of the $\delta$-function, $\sim \delta\left(\omega_{1}-E_{0}\right)$, takes off the energy dependence in the probabilities like Eq. 7, so that the transition probabilities and the polarization vector Eq. 2 depend on the fundamental vacuum constants, $\Delta m^{2}, s_{2}$ and on the magnetic field parameter $\mu B_{\perp}$ which is changing very slowly comparing with the event counting at the underground detectors. Use of $\delta\left(\omega_{1}-E_{0}\right)$-function means also that we have neglected the thermal and Doppler broadening of the beryllium line [21]. As result in the case of the beryllium neutrinos we can parameterize the recoil electron spectra over the values $P=P\left(\mu B_{\perp}\right)$ and $A=A\left(\mu B_{\perp}\right)$ independently on neutrino energy as well as for the Dirac neutrino conversions $\nu_{e_{L}}^{(D)} \rightarrow \nu_{e_{R}}^{(D)}$ [14] which do not depend on neutrino energy at all.

\subsection{Scattering of Majorana and Dirac neutrinos off electrons}

Now using the spin density matrices Eq. (4), Eq. (5) with the given polarization components Eq. (2), Eq. (6) let us consider what kind of differences of the Majorana and Dirac neutrino scattering off electrons can arise in underground experiments.

The key argument here is the difference of the matrix element for the Majorana neutrino current ,

$$
\begin{aligned}
<k_{2} r_{2}\left|N\left(\hat{\bar{\Psi}}^{(M)} \gamma_{\mu} \frac{\left(1-\gamma_{5}\right)}{2} \hat{\Psi}^{(M)}\right)\right| k_{1}, r_{1}>=\quad & -\bar{u}^{r_{2}}\left(k_{1}\right) \gamma_{\mu} \gamma_{5} u^{r_{1}}\left(k_{1}\right)= \\
& =\bar{v}^{r_{1}}\left(k_{1}\right) \gamma_{\mu} \gamma_{5} v^{r_{2}}\left(k_{2}\right)
\end{aligned}
$$


from the corresponding one for the Dirac neutrino $\left(\bar{u}^{r_{2}}\left(k_{2}\right)\left(\gamma_{\mu}\left(1-\gamma_{5}\right) / 2\right) u^{r_{1}}\left(k_{1}\right)\right)$ or for the Dirac antineutrino $\left(\bar{v}^{r_{1}}\left(k_{1}\right)\left(\gamma_{\mu}\left(1-\gamma_{5}\right) / 2\right) v^{r_{2}}\left(k_{2}\right)\right)$.

Absence of the vector part in the matrix element Eq. (8) is crucial for the neutrino interactions with particles in the case of partial polarization, and it is the direct consequence of the charge-conjugation property $C \hat{\Psi}^{(M)} C^{-1}=\hat{\Psi}^{(M)}$ for the secondquantized Majorana field[22]

$$
\hat{\Psi}^{(M)}(x)=\sum_{\vec{p} r} \frac{1}{\sqrt{2 E_{p}}}\left\{c_{\vec{p}}^{r} u^{r}(p) e^{-i p x}+c_{\vec{p}}^{r+} v^{r}(p) e^{i p x}\right\} .
$$

The matrix element for the $\nu e$-scattering $M=M_{\text {weak }}+M_{e m}$ consists of the two terms for a Majorana neutrino,

$$
\begin{aligned}
M_{\text {weak }}\left(\nu_{i} e^{-} \rightarrow \nu_{i} e^{-}\right)= & -2 G_{F} \sqrt{2}\left[\bar{u}^{r_{2}}\left(k_{2}\right) \gamma^{\alpha} \gamma_{5} u^{r_{1}}\left(k_{1}\right)\right] \times \\
\times & {\left[\bar{u}_{e}\left(p_{2}\right)\left(g_{i L} \gamma_{\alpha} \frac{\left(1-\gamma_{5}\right)}{2}+g_{R} \gamma_{\alpha} \frac{\left(1+\gamma_{5}\right)}{2}\right) u_{e}\left(p_{1}\right)\right], }
\end{aligned}
$$

and

$$
M_{e m}=\frac{i e}{q^{2}}\left[\bar{u}^{r_{2}}\left(k_{2}\right)\left(\mu_{12}^{(M)}-i d_{12}^{(M)} \gamma_{5}\right) \sigma_{\alpha \beta} q^{\beta} u^{r_{1}}\left(k_{1}\right)\right]\left[\bar{u}_{e}\left(p_{2}\right) \gamma^{\alpha} u_{e}\left(p_{1}\right)\right] .
$$

Here $q_{\beta}=\left(k_{2}-k_{1}\right)_{\beta}$ is the momentum transfer; $g_{i L}=\xi \pm 0.5$ and $g_{R}=\xi$ are the constants of the standard model with the Weinberg angle parameter $\xi=\sin ^{2} \theta_{W}=$ 0.23 , where upper (lower) sign for $g_{i L}$ corresponds to the subscript $i=e$ for electron neutrino ( $i=\mu$ for muon neutrinos).

We have meant here that in the presence of a flavor mixed neutrino flux from the Sun described by the spin density matrices (41), (5) the $\nu_{i} e \rightarrow \nu_{i} e$ - scattering weak amplitude $M_{\text {weak }}$ conserving helicity (= chiralities L or R) adds the coherent electromagnetic amplitude $M_{e m}$ of the spin-flavor transition $\nu_{\mu_{L}, R} e \rightarrow \nu_{e_{R}, L} e$ in the case of electron neutrinos $(i=e)$ or of the inversed process $\nu_{e_{L}, R} e \rightarrow \nu_{\mu_{R},{ }_{L}} e$ in the case of muon neutrinos, $i=\mu$.

Notice that we do not distinguish the mass eigen-states in the electromagnetic scattering $\nu_{1} e \rightarrow \nu_{2} e$ through the transition dipole moments $\mu_{12}, d_{12}$ from the flavor states in the weak amplitude Eq. (10) because of the inclusion of mixing angles into $\mu_{12}, d_{12}$ and the ultrarelativistic approximation used in spinors. Thus, we put $m_{1}=m_{2}=m_{\nu_{e}}=0$ elsewhere except for $\mu_{12}, d_{12}$ themselves or even do not bother about the limit $m_{\nu} \rightarrow 0$ for a large moment $\mu \sim 10^{-10} \mu_{B}$ in some known extended models where $\mu_{i j}$ does not depend on neutrino masses.

It is useful to retain $\gamma_{5}$ in CP-violating term in Eq. (11) $\left(\sim d_{12}\right)$ without the obvious change $\gamma_{5} u^{r_{1}}=r_{1} u^{r_{1}}$ for the identical chirality and helicity (in ultrarelativistic approximation used) because of the mixed polarization Eq. (4), or due to an arbitrary $r_{1}$. 
Using Eq. (41), Eq. (10), Eq. (11) we obtain the differential cross-section for the Majorana neutrino scattering off electrons,

$$
\frac{d \sigma^{(M)}}{d T d \phi}=\left(\frac{d \sigma^{(M)}}{d T d \phi}\right)_{w e a k}+\left(\frac{d \sigma^{(M)}}{d T d \phi}\right)_{e m}+\left(\frac{d \sigma^{(M)}}{d T d \phi}\right)_{i n t}
$$

where the weak amplitude Eq. (10) leads to the sum of contributions of $\nu_{e} e-$ and $\nu_{\mu} e$ -scattering

$$
\begin{aligned}
\left(\frac{d \sigma^{(M)}}{d T d \phi}\right)_{w e a k}= & \frac{G_{F}^{2} m_{e}}{\pi^{2}}\left\{P\left[g_{e L}^{2}+g_{R}^{2}\left(1-\frac{T}{\omega_{1}}\right)^{2}-\frac{m_{e} T}{\omega_{1}^{2}} g_{e L} g_{R}\right]+\right. \\
& +(A-P)\left[g_{R}^{2}+\left(1-\frac{T}{\omega_{1}}\right)^{2} g_{e L}^{2}-\frac{m_{e} T}{\omega_{1}^{2}} g_{e L} g_{R}\right]+ \\
& +P_{1}\left[g_{\mu L}^{2}+g_{R}^{2}\left(1-\frac{T}{\omega_{1}}\right)^{2}-\frac{m_{e} T}{\omega_{1}^{2}} g_{\mu L} g_{R}\right]+ \\
& \left.+\left(1-A-P_{1}\right)\left[g_{R}^{2}+\left(1-\frac{T}{\omega_{1}}\right)^{2} g_{\mu L}^{2}-\frac{m_{e} T}{\omega_{1}^{2}} g_{\mu L} g_{R}\right]\right\} .
\end{aligned}
$$

and the electromagnetic amplitude Eq. (11) gives

$$
\left(\frac{d \sigma^{(M)}}{d T d \phi}\right)_{e m}=\frac{\alpha^{2}}{2 m_{e}^{2}}\left(\frac{\mu_{12}^{2}+d_{12}^{2}}{\mu_{B}^{2}}\right)\left[\frac{1}{T}-\frac{1}{\omega_{1}}\right] .
$$

Both these terms in Eq. (12) do not depend on the azimuthal angle $\Phi$. However, the last interference term in Eq. (12),

$$
\begin{aligned}
& \left(\frac{d \sigma^{(M)}}{d T d \phi}\right)_{i n t}=\frac{\alpha G_{F}}{4 \sqrt{2} \pi m_{e} T} \times \\
& \times\left\{( \frac { \mu _ { 1 2 } } { \mu _ { B } } ) \left[\left(g_{e L}+g_{\mu L}+2 g_{R}\right)\left(\overrightarrow{p_{2}} \cdot\left(\vec{\xi}_{\perp}^{(\mu \tilde{e})}+\vec{\xi}_{\perp}^{(e \tilde{\mu})}\right)\right)\left(2-\frac{T}{\omega_{1}}\right)+\right.\right. \\
& \left.+\left(g_{\mu L}-g_{e L}\right)\left(\overrightarrow{p_{2}} \cdot\left(\vec{\xi}_{\perp}^{(\mu \tilde{e})}-\vec{\xi}_{\perp}^{(e \tilde{\mu})}\right)\right) \frac{T}{\omega_{1}}\right]- \\
& -\left(\frac{d_{12}}{\mu_{B}}\right)\left[( g _ { e L } + g _ { \mu L } + 2 g _ { R } ) \left(\hat{\vec{k}}_{1} \cdot\left[\vec{p}_{2} \times\left(\vec{\xi}_{\perp}^{(\mu \tilde{e})}+\vec{\xi}_{\perp}^{(e \tilde{\mu})}\right)\right)\left(2-\frac{T}{\omega_{1}}\right)+\right.\right. \\
& \left.\left.+\left(g_{\mu L}-g_{e L}\right)\left(\hat{\vec{k}}_{1} \cdot\left[\vec{p}_{2} \times\left(\vec{\xi}_{\perp}^{(\mu \tilde{e})}-\vec{\xi}_{\perp}^{(e \tilde{\mu})}\right)\right]\right) \frac{T}{\omega_{1}}\right]\right\},
\end{aligned}
$$

depends on that angle via the sum in braces, or the cross-section can be rewritten as the product

$$
\left(\frac{d \sigma^{(M)}}{d T d \phi}\right)_{i n t}=\frac{\alpha G_{F}}{4 \sqrt{2} \pi m_{e} T}\left|\vec{p}_{2}\right| \sin \theta_{p_{2} k_{1}}\left[\left(\frac{\mu_{12}}{\mu_{B}}\right) \cos \phi+\left(\frac{d_{12}}{\mu_{B}}\right) \sin \phi\right] \times
$$




$$
\begin{aligned}
& \times\left[\left(g_{e L}+g_{\mu L}+2 g_{R}\right)\left(2-\frac{T}{\omega_{1}}\right)\left(\left|\vec{\xi}_{\perp}^{(\mu \tilde{e})}+\vec{\xi}_{\perp}^{(e \tilde{\mu})}\right|\right)+\right. \\
& \left.+\left(g_{\mu L}-g_{e L}\right) \frac{T}{\omega_{1}}\left(\left|\vec{\xi}_{\perp}^{(\mu \tilde{e})}-\vec{\xi}_{\perp}^{(e \tilde{\mu})}\right|\right)\right],
\end{aligned}
$$

where $\left|\vec{p}_{2}\right| \sin \theta_{p_{2} k_{1}}=\sqrt{2 m_{e} T\left(1-T / T_{\max }\right)}, T_{\max }=2 \omega_{1}^{2} /\left(m_{e}+2 \omega_{1}\right)$ is the maximum kinetic energy of the recoil electron, and the transversal polarization values Eq. (6), $\left|\vec{\xi}_{\perp}^{(\mu \tilde{e})}\right|=2 \sqrt{P_{1}(A-P)},\left|\vec{\xi}_{\perp}^{(e \tilde{\mu})}\right|=2 \sqrt{P\left(1-A-P_{1}\right)}$, are the external parameters which are given by a neutrino dynamics within solar interior.

The polarization vectors $\vec{\xi}_{\perp}^{(a)}$ should follow the solar magnetic field direction at the resonant point along neutrino trajectory obeying the extremum conditions $d \xi_{z}^{(\mu \tilde{e})} / d t \equiv$ $d\left(A-P-P_{1}\right) / d t=0, d \xi_{z}^{(e \tilde{\mu})} / d t \equiv d\left(1-A-P-P_{1}\right) / d t=0$ or $d \xi_{z}^{(0 e)} / d t \equiv d(A-$ $2 P) / d t=0$, and the Bargman-Mishel-Telegdi' equation for the electroneutral fermion spin motion in the external magnetic field $\mathbf{B} 23$,

$$
\frac{d \xi_{z}^{(a)}}{d t}=2 \mu_{\nu}\left(\vec{\xi}_{\perp}^{(a)} \cdot[\vec{n} \times \vec{B}]\right)
$$

Here the unit vector $\mathbf{n}=\mathbf{k}_{1} / \omega_{1}$ and the neutrino momentum $\mathbf{k}_{1}=\left(0,0, \omega_{1}\right)$ are orthogonal to the transversal polarization, $\left(n_{i} \cdot \xi_{\perp i}^{(a)}\right)=0$, and $a=(0 e),(0 \mu),(\mu \tilde{e}),(e \tilde{\mu})$ is the flavor index. Since all transversal polarization vectors are aligned along the magnetic field $\mathbf{B}$ we may change the modulus of the vector difference and sum $\left|\vec{\xi}_{\perp}^{(\mu \tilde{e})} \pm \vec{\xi}_{\perp}^{\left.e^{e} \tilde{\mu}\right)}\right|$ in the interference cross-section (16) to the difference and sum of the vector values $\left.\left|\vec{\xi}_{\perp}^{(\mu \tilde{e})}\right| \pm \mid \vec{\xi}_{\perp}^{\tilde{\mu}}\right) \mid$ given by Eq. ([) .

It is obvious that the interference term (15) vanishes for the separated electron $\left(A=1, P_{1}=0\right)$ and muon $(A=P=0)$ systems.

Let us recall that, in general, the electron neutrino survival probability $P=$ $P\left(\omega_{1}, \mu B_{\perp}, s_{2}, \Delta m^{2}\right)$, the muon neutrino parameter $P_{1}=P_{1}\left(\omega_{1}, \mu B_{\perp}, s_{2}, \Delta m^{2}\right)$ and the normalization factor $A=A\left(\omega_{1}, \mu B_{\perp}, s_{2}, \Delta m^{2}\right)$ appearing in Eq. (13) Eq. (15), are some functions of the incident neutrino energy $\omega_{1}$, of the solar magnetic field $B_{\perp}$ multiplied by $\mu$, and of the fundamental vacuum parameters $s_{2}=\sin 2 \theta, \Delta m^{2}=$ $m_{1}^{2}-m_{2}^{2}$.

Analogously to Eq. (14) which is proportional to the factor $\left(\mu_{12}^{2}+d_{12}^{2}\right)$ in the interference cross section Eq. (15) both terms that are linear in $\mu_{12}$ and $d_{12}$ have the same CP-signs while the intrinsic CP-signs of these dipole moments are opposite. Actually, in Eq. (15) this coincidence of CP-signs is due to the different CP-signs of the kinematic factors before transition moments, or due to the presence of the third additional 3-vector $\hat{k}_{1}$ before $d_{12}$. On the other hand, if CP-holds: $(C P) \mathcal{L}_{\text {int }}(C P)^{-1}=$ $\mathcal{L}_{\text {int }}$, one term survives in Eq. (14) and Eq. (15) only: either $\sim \mu_{12}$ or $\sim d_{12}$.

Notice that for the case $d_{12}=0$ the cross-section Eq. (12) coincides after a change of notations with the recent result [24] obtained by I.V. Gaidaenko.

Let us compare the result Eq. (12) with the analogous Eq.(11) obtained in 14 for the Dirac neutrino conversions $\nu_{e_{L}} \leftrightarrow \nu_{e_{R}}$ in the Sun with the following neutrino 
scattering off electrons in underground detectors. The electromagnetic term Eq. (14) is the same as in the Dirac case with the trivial change $\mu_{\nu}^{(D)} \rightarrow \sqrt{\mu_{12}^{2}+d_{12}^{2}}$ for a Majorana neutrino. However, the main term, Eq. (13), and the interference one Eq. (15) have electron energy spectra which are different from the spectra for the corresponding terms in [14].

Really, repeating calculations of [14] with use of Eq. (4) for the separated $\nu_{e_{L}}, \nu_{e_{R}}{ }^{-}$ system $(A=1)$ we find

$$
\left(\frac{d \sigma^{(D)}}{d T d \phi}\right)_{\text {weak }}=P \frac{G_{F}^{2} m_{e}}{\pi^{2}}\left[g_{L}^{2}+g_{R}^{2}\left(1-\frac{T}{\omega_{1}}\right)^{2}-\frac{m_{e} T}{\omega_{1}^{2}} g_{L} g_{R}\right],
$$

and

$$
\left(\frac{d \sigma^{(D)}}{d T d \phi}\right)_{i n t}=-\left(\overrightarrow{p_{2}} \cdot \vec{\xi}_{\perp}^{(0 e)}\right) \frac{\mu_{\nu}^{(D)}}{\mu_{B}} \frac{\alpha G_{F}}{2 \sqrt{2} \pi m_{e} T}\left(g_{L}+g_{R}\left[1-\frac{T}{\omega_{1}}\right]\right),
$$

where the last interference term is exactly Eq. (11c) in [14], but the weak interaction term Eq. (17) is two times bigger than Eq. (11a) in [14.

Our cross-sections $\left(d \sigma^{(M, D)} / d T\right)_{\text {weak }}$ Eq. (13), Eq. (17) coincide with the standard results [25] for the scattering of the massless, fully-polarized neutrinos. For instance, for the separated $\nu_{e_{L}}, \tilde{\nu}_{e_{R}}$-system $(\mathrm{A}=1)$ this coincidence, $\left(d \sigma^{(M)} / d T\right)_{\text {weak }}=$ $\left.d \sigma^{(D)} / d T\right)_{\text {weak }}$, is shown in Fig. 1 by the common line "a" which describes both the cross section of the $\nu_{e} e \rightarrow \nu_{e} e$-scattering for the left-handed Majorana neutrinos with the substitution $\xi_{z}^{(0 e)}=-1(\mathrm{~A}=\mathrm{P}=1)$ to Eq. (13) and the cross section Eq. (17) for the fully-polarized Dirac neutrinos with the substitution $\mathrm{P}=1$.

One can also check from Eq. (13) that the right-handed fully polarized Majorana neutrinos, $\xi_{z}^{(0 e)}=1, \mathrm{~A}=1, \mathrm{P}=0$, are described by the standard cross-section for the Dirac antineutrinos $\left(g_{L} \leftrightarrow g_{R}\right.$ and $P \rightarrow 1$ in Eq. (17) which, in contrast to the Majorana $\tilde{\nu}_{e_{R}}$, can not be observed in solar neutrino flux (line "d" in Fig. 1).

Moreover, in the particular case $A=1$ and after a trivial transition from CM to the laboratory frame of reference we have found the full coincidence of our Eq. (13) with the old result Eq. (3.9) in [26].

If a sharp difference in the slope of lines for the Majorana neutrinos ("b", "c", "d" lines in Fig.1) and for the Dirac ones ("e", "f") was marked in the Borexino or Hellaz experiment this would be a test whether the solar neutrinos are the Majorana particles or they are the Dirac ones. It does not matter which concrete value of unknown parameter $\mu B_{\perp}$ influencing the survival probability $P$ occurs within the Sun: all corresponding lines given in Fig.1 for the same probabilities (pairs ("b", "e") and ("c", "f")) have quite different slopes.

Emphasize that we have simplified the observable averaged cross sections $<d \sigma / d T>$ using $\delta\left(\omega_{1}-E_{0}\right)$-function for the beryllium neutrinos. All lines are interrupted at $T_{\max } \simeq 0.663 \mathrm{keV}$ (see also Fig.2,3) and authors 18] are planning to distinguish the "signature edge" of the recoil spectrum from the monoenergetic Be neutrino flux. 
This difference appears also for a non-separated system (merging of resonances in the mechanism[7]) shown in Fig. 2 for the particular equipartition case $P=\nu_{e_{L}}^{*} \nu_{e_{L}}=$ $\tilde{\nu}_{e_{R}}^{*} \tilde{\nu}_{e_{R}}=\nu_{\mu_{L}}^{*} \nu_{\mu_{L}}=\tilde{\nu}_{\mu_{R}}^{*} \tilde{\nu}_{\mu_{R}}=0.25$ when the $\nu_{\mu} e$-scattering is taken into account.

The similar difference between Majorana and Dirac neutrino cases can be found from the total cross section behavior shown in Fig.3 for the separated electron neutrino system $(\mathrm{A}=1)$. These cross sections $\sigma\left(W_{e}\right)$ contribute to the total number of neutrino events which can be observed in some different energy bins separated by the different thresholds $W_{e}$. For a low energy region all lines depending on the different parameters $\mathrm{P}$ which correspond to the Majorana neutrino cross sections become parallel to each other (lines "b", "c" in Fig. 3) but the analogous Dirac neutrino cross-sections (lines "d", "e" with the same P as in the Majorana case) change their slope. Thus, if the magnetic field value $B_{\perp}$ in the Sun is somehow changing and, therefore, influences the total cross section $\sigma_{\nu e}^{(D, M)}\left(W_{e}\right)$ (i.e. leads to the transition "b" $\rightarrow$ "c", or " d" $\rightarrow$ "e") we can distinguish Majorana and Dirac neutrinos due to different slopes of the event spectra over $W_{e}, N_{\nu}\left(W_{e}\right) \sim<\sigma_{\nu e}^{(D, M)}\left(W_{e}\right)>$.

The resonant spin precession can appear for another changing magnetic field configuration, for instance, for the linear-polarized Alfven wave [27] when a magnetic field direction (transversal to the incident neutrino momentum) could be fixed. However, in contrast to [6, 7], this mechanism is still not elaborated for $\nu_{e_{L}} \rightarrow \tilde{\nu}_{\mu_{R}}$ and $\nu_{e_{L}} \rightarrow \tilde{\nu}_{e_{R}}$-conversions.

\section{Discussions and conclusions}

The partially polarized electron neutrino flux provided by $\nu_{e_{L}} \rightarrow \tilde{\nu}_{e_{R}}$ conversions within the Sun can give an opportunity to distinguish Majorana and Dirac neutrinos in the Borexino and in the Hellaz experiments due to quite different profiles of recoil electron spectra(Fig.1-3). Notice that measurements of $T$ and $\theta_{p_{2} k_{1}}$ would allow authors of the project [19] to determine the neutrino energy $\omega_{1}$.

The inclusion of other neutrino fluxes with the different $\Phi_{i}\left(\omega_{1}\right)$ is necessary for comparison with real electron spectra although we expect the same qualitative difference of the spectra for the Majorana and the Dirac cases.

Really, it is obvious that $d \sigma^{(M)} / d T$ exceeds $d \sigma^{(D)} / d T$ in a wide energy region due to additional interaction of right-handed active Majorana neutrinos with electrons in contrast to the sterile $\nu_{R}^{(D)}$.

Probably, in the Superkamiokande or in the SNO detectors this difference can be marked too. In spite of unknown dependence of the survival probability $P\left(\omega_{1}, \mu B_{\perp}\right)$ on the incident neutrino energy $\omega_{1}$ both the differential $\langle d \sigma / d T\rangle$ and the total $\left\langle\sigma\left(W_{e}\right)\right\rangle$ cross-sections averaged over the boron neutrino spectrum $\lambda\left(\omega_{1}\right)$ [21] should have quite different slopes depending on $T$ or $W_{e}$. One can easily check this statement substituting a fixed probe value of $0 \leq P<1$ and integrating Eq. (13), 
Eq. (17) over the spectrum $\lambda\left(\omega_{1}\right)$ [21]. The absolute value of the averages $\langle d \sigma / d T\rangle$, $<\sigma\left(W_{e}\right)>$ changes when $P$ varies that corresponds to some different levels of the electron neutrino deficit. Meanwhile both sets of lines (for Majorana or Dirac neutrinos) conserve different slopes for the two kinds of particles.

Another model independent way to distinguish Majorana and Dirac neutrinos was proposed in [28]. Authors 28] showed that NC events at the SNO detector would not oscillate over time for $\nu^{(M)}$ and would oscillate in the case of $\nu^{(D)}$. The method is based on any active-active transitions $\nu_{a} \rightarrow \tilde{\nu}_{b}$ in the solar magnetic field for Majorana neutrinos and on active-sterile conversions in the Dirac case. A changing magnetic field $B(t) \neq 0$ influences neutrino flux variations so that one predicts $d N^{N C} / d t=0$ for $\nu^{(M)}$ and $d N^{N C} / d t \neq 0$ for $\nu^{(D)}$.

While in the method [28] one expects $d N^{N C} / d t=0$ for both kinds of neutrinos if $B \simeq$ const, in our method in a constant magnetic field the profiles $<d \sigma^{(M)} / d T>$ and $<d \sigma^{(D)} / d T>$ remain different.

In conclusion, without any knowledge of solar magnetic field direction and even for a magnetic moment obeying the astrophysical constraint $\mu \lesssim 3 \times 10^{-12} \mu_{B}$ [8] one can distinguish Majorana and Dirac neutrino measuring the recoil electron spectra in the low energy $\nu_{e} e$-scattering in underground detectors and comparing slopes of their profiles.

Last remark concerns a possibility to observe some variations of neutrino flux in detectors with large statistics of events. Let us assume that the unknown parameter $\mu B_{\perp}$ can change somehow in the Sun influencing $P\left(\mu B_{\perp}\right)$. In this case the spectra $<d \sigma^{(M)} / d T>$ will be displaced parallel to the initial one without change of a slope in the contrary to the spectra $\left\langle d \sigma^{(D)} / d T>\right.$ that should change its slope. This behaviour of spectra would be a strong argument in favour of the mechanism[7] as solution of the solar neutrino problem.

\section{Acknowledgment}

I acknowledge George Zatsepin for useful discussion and for pointing out Refs. [18], [19] and correspondence with Robert Shrock and Samoil Bilenky regarding the Refs.

[2], [26] and the Ref. [28]. I am grateful to I.V. Gaidaenko who was first obtaining correct cross-section of the $\nu e$-scattering of partially polarized Majorana neutrinos and indebted to him for sending of his preprint [24] that allowed me to improve a wrong previous version of Eq. (12). This work was supported in part from RFFR under grants N. 95-02-03724 and N. 97-02-16501.

\section{References}

[1] R. Davis, Phys. Rev. 97 (1955) 766.

[2] K. Fujikawa, R.E. Shrock, Phys. Rev. Lett. 45 (1980) 963. 
[3] J. Schechter, J.W.F. Valle, Phys. Rev. D 24 (1981) 1883; Err. Phys. Rev. D 25 (1982) 283.

[4] M.B. Voloshin, M.I. Vysotsky, and L.B. Okun', Zh. Eksp. Theor. Fiz., 91 (1986) 754. (Sov. Phys. JETP, 64 (1986) 446).

[5] S. Mikheyev and A. Smirnov, Sov. J. Nucl. Phys. 42 (1986) 913;

L. Wolfenstein, Phys. Rev. D 17 (1978) 2369; ibid. D 20 (1979) 2634.

[6] E. Kh. Akhmedov, Phys. Lett. B 213 (1988) 64; C.-S. Lim and W.J. Marciano, Phys. Rev. D 37 (1988) 1368.

[7] E.Kh. Akhmedov, S.T. Petcov and A.Yu. Smirnov, Phys. Rev. D 48 (1993) 2167.

[8] G. Raffelt and A. Weiss, Astron. Astrophys., 264 (1992) 536. Georg G. Raffelt, Stars as Laboratories for Fundamenental Physics (the University of Chicago Press, Chicago-London, 1996) p 279.

[9] R. Barbieri and R.N. Mohapatra, Phys. Rev. Lett., 61 (1988) 27.

[10] A. Kuldjiev, Nucl. Phys. B 243 (1984) 387. G.S. Vidyakin, Pis'ma Zh. Eksp. Theor. Fiz. 55 (1992) 212. (JETP Lett. 55 (1992) 206).

[11] D.W. Hughes, in: Advances in solar system magnetohydrodynamics (ed. by E.R. Priest, A.W. Hood, Cambridge University Press, 1991) Fig. 5.1.

[12] V.A. Kutvitskii and L.S. Solov'ev, Zh. Eksp. Theor. Fiz. 105 (1994) 853. (Sov. Phys. JETP, 78 (1995) 456).

[13] S. Pastor, V. Semikoz and J.W.F. Valle, Phys. Lett., B369 (1996) 301.

[14] R. Barbieri, G. Fiorentini, Nucl. Phys. B 304 (1988) 909;

P. Vogel, J. Engel, Phys. Rev. D 39 (1989) 3378.

[15] Z.G. Berezhiani, M. Moretti, and A. Rossi, Z. Phys. C 58 (1993) 423.

[16] A.Yu. Smirnov, Phys. Lett. B 260 (1991) 161.

[17] Y. Suzuki, (1993) in: Proc. Int. Symposium on Neutrino Astrophysics, (Takayama Kamioka, Japan, Oct. 19-22, 1992)

[18] R.S. Raghavan, Science 267 (1995) 45.

[19] F. Arzarello et al. Preprint CERN-LAA/94-19, College de France LPC/94-28 (1994). 
[20] S.M. Bilenky, Introduction in Feynmann diagrams and Physics of the Electroweak Interaction (Energoatomizdat (in Russian), Moscow, 1990) formula (D.54). Translated in Basics of Physics, Frontiers (1994).

[21] John N. Bahcall, Neutrino Astrophysics ( Cambridge University Press, 1988) section 6.3.

[22] E.D. Commins, P.H. Buksbaum, Weak Interactions of leptons and quarks ( Cambridge University Press, 1983) section 10.4 .

[23] V.B. Berestetski, E.M. Lifshits, and L.P. Pitaevski, Quantum Electrodynamics, 2nd ed. (Pergamon Press, Oxford, 1982).

[24] I.V. Gaidaenko, Preprint ITEP, ITEP-15/97

[25] L.B. Okun', Leptons and Quarks, (North-Holland, Amsterdam, 1982).

[26] B. Kayser and R.E. Shrock, Phys. Lett. B 112 (1982) 137.

[27] V.B. Semikoz, Phys. Rev. D48 (1993) 5264.

[28] S.M. Bilenky and C. Giunti, Preprint DFTT 75/93, hep-ph/9312211 (1993) 


\section{Figure Captions}

Fig. 1. The differential spectra Eqs. (13) and (17) for the beryllium neutrino in the case of the separated $\nu_{e_{L}}, \tilde{\nu}_{e_{R}}$-system (the normalization parameter A in Eq. (3) equals to unity, $A=1)$ :

The lines "b", "c", "d" correspond to an incident Majorana neutrino with the survival probability $P=0.5,1 / 3,0$ correspondingly, and the lines "e", "f" describe cross sections for a Dirac neutrino in the cases $P=0.5,1 / 3$. The line "a" is the common one for Majorana and Dirac fully-polarized left-handed neutrinos with $P=1$.

Fig. 2. The same spectra in the case of the equipartition $P=\nu_{e_{L}}^{*} \nu_{e_{L}}=\tilde{\nu}_{e_{R}}^{*} \tilde{\nu}_{e_{R}}=$ $\nu_{\mu_{L}}^{*} \nu_{\mu_{L}}=\tilde{\nu}_{\mu_{R}}^{*} \tilde{\nu}_{\mu_{R}}=0.25$, the parameter $A=0.5$ :

The line "a" describes Majorana neutrino, the line "b"- Dirac neutrino.

Fig. 3. The total cross sections for the beryllium neutrino $\left\langle\sigma^{(M, D)}\left(W_{e}\right)>\right.$ in dependence on the threshold energy $W_{e}$. For a liquid scintillator it assumes a low threshold $W_{e}$ of order $\sim$ a few hundreds keV. The line "a" corresponds to the fully polarized left-handed neutrinos $(P=1)$ with $<\sigma^{(M)}\left(W_{e}\right)>=<\sigma^{(D)}\left(W_{e}\right)>$ (SSM prediction without $\nu_{e}(B e)$ - deficit); lines "b", "c" are plotted for Majorana neutrino with the survival probabilities $P=0.5$ and $P=1 / 3$ correspondingly; lines "d", "e" are plotted for Dirac neutrino with the same $P=0.5$ and $P=1 / 3$. 


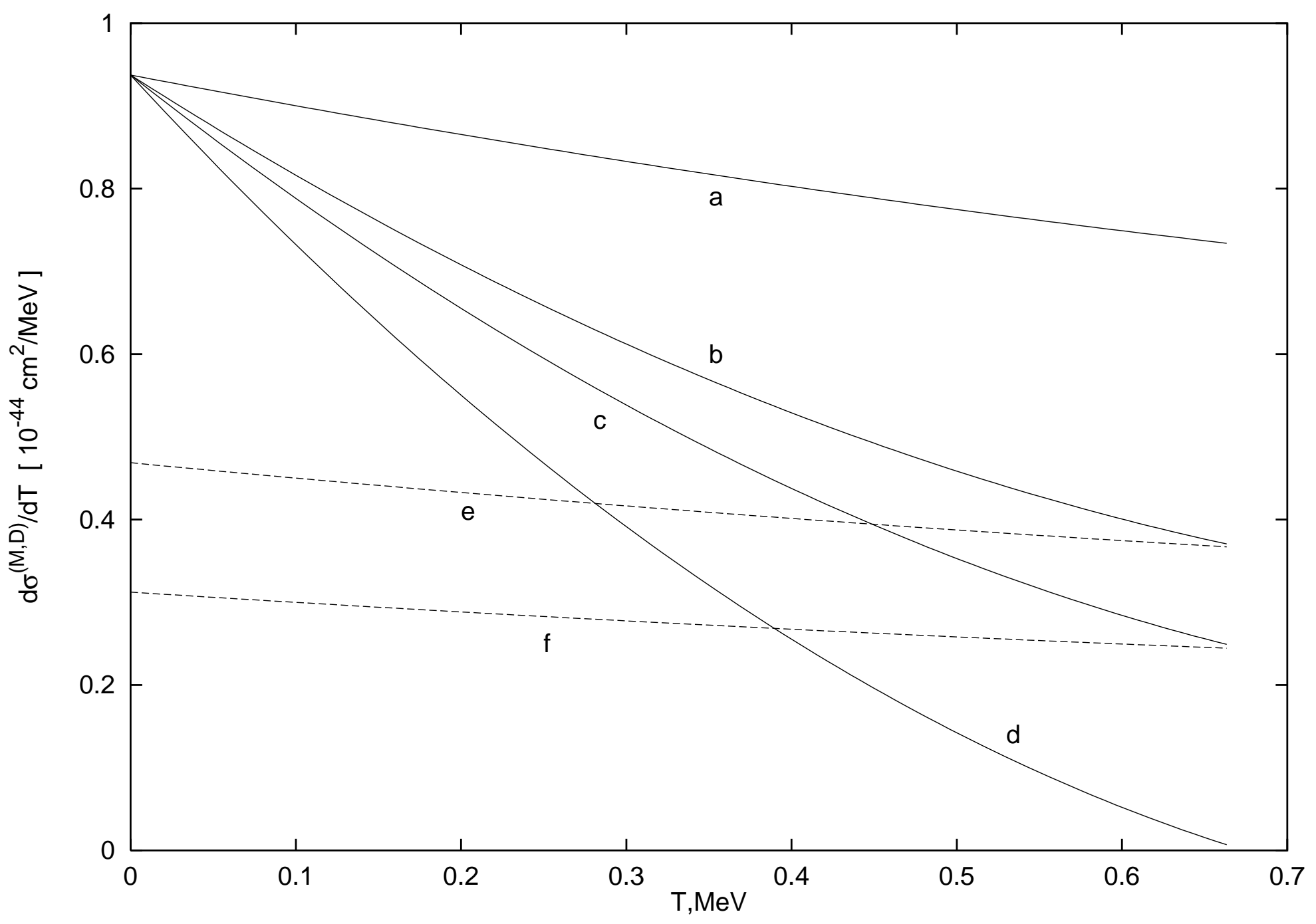




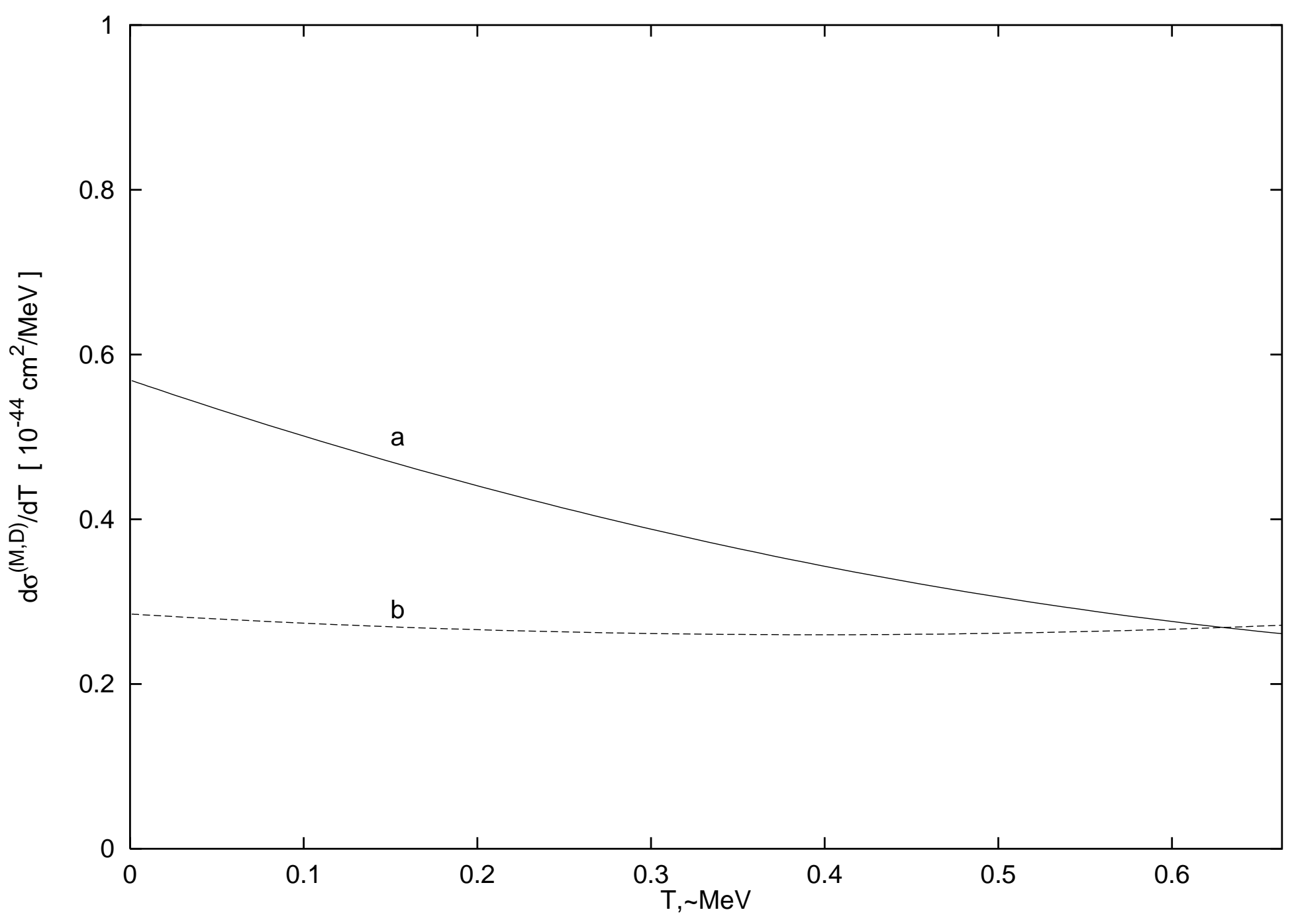


